\title{
EEG microstate architecture does not change during passive whole-body accelerations
}

\author{
M. Ertl $^{1,2}$ (1) $\cdot$ M. Schulte ${ }^{1}$ M. Dieterich ${ }^{1,3,4}$
}

Received: 6 February 2020 / Revised: 10 March 2020 / Accepted: 13 March 2020 / Published online: 28 May 2020

(c) The Author(s) 2020

\section{Dear Sirs,}

EEG microstates are defined as brief periods during which the overall scalp topography remains stable. Only four distinct microstates, explaining up to $80 \%$ of the variance in rest EEG, were consistently found in microstate studies [1,2]. Additionally, an association between the EEG microstates and resting state fMRI likely exists [3, 4]. The link between the four canonical microstates (A, B, C, D) and sensory manipulations has been addressed, but a coherent theory has not yet emerged. For example, the temporal parameters of microstate $\mathrm{B}$ could be altered by manipulation of the visual input [5].

The link between microstate B and the visual system [5] motivated us to investigate whether an association exists between one of the microstates and vestibular stimulation. This is of special interest, since a close interaction between the different sensory systems is known from fMRI studies [6], e.g., showing a change of the interaction mode with the shift of the dominance from one system to the other for the visual and somatosensory systems [7]. Compared to other sensory systems, the vestibular system possesses a few unique features. For example, natural stimulation of the system always causes a multimodal stimulation of multiple sensory systems [8]. Vestibular information is processed by a distributed cortical network represented in both

M. Ertl

matthias.ertl@med.uni-muenchen.de

1 Department of Neurology, Ludwig-Maximilians-Universität München, Marchioninistraße 15, 81377 Munich, Germany

2 Department of Psychology, University of Bern, Bern, Switzerland

3 German Center for Vertigo and Balance Disorders (DSGZ), Ludwig-Maximilians-Universität München, Munich, Germany

4 Munich Cluster for Systems Neurology (SyNergy), Munich, Germany hemispheres [9-11] with a preponderance in the non-dominant hemisphere [12]. Additionally, a reciprocal inhibitory interaction between the vestibular and the visual system was demonstrated [13].

Here, we investigated whether passive whole-body movements with weak to moderate acceleration intensities influence the overall microstate architecture in healthy participants sitting on a chair on a motion platform. The motivation for the experiment was to test whether passive body accelerations, which are mostly but not solely [8] sensed by vestibular input, have a similar impact on microstates as visual input.

The EEGs of 29 healthy volunteers (12 female, 17 male; 26.7 years $\pm 5.59 \mathrm{SD}$ ) were analyzed during passive body translations along the three main axes (fore/aft, left/ right, up/down) generated by a motion platform (Moog@6DOF2000E) and compared to the static rest condition. Sinusoidal profiles with a frequency of $0.5 \mathrm{~Hz}$ and an amplitude of $3 \mathrm{~cm}$ were used. The stimulation duration along every axis was $35 \mathrm{~s}$. Subjects were instructed to keep their eyes closed and stay awake.

The microstate analysis was performed in Matlab (Mathworks) using the EEGlab plug-in MicrostateAnalysis (Version 0.3, Thomas König). The data were band-pass filtered $(2-20 \mathrm{~Hz})$ and visually inspected for artifacts. Segments contaminated with artifacts were removed. The data were clustered within subjects using the widely used 'atomize and agglomerate hierarchical clustering' (AAHC) algorithm [14] and by ignoring polarity. Averages across subjects were calculated for any of the four movement conditions (Fig. 1), and a grand average across the conditions was computed. The statistical values for duration, occurrence, contribution, and explained variance were extracted and a between condition ANOVA was calculated for any of the four movement conditions.

The microstates obtained for the four conditions resembled the spatial distributions of the four canonical microstates and showed high inter-condition similarities (Fig. 1). 
Fig. 1 The topographies (head seen from above, nose up, left ear on the left) of the four microstate types (A-D) retrieved from the clustering algorithm for the four different motion conditions (static; fore/ aft; left/right; up/down). The four types resemble the topographies reported by other studies and were sorted accordingly

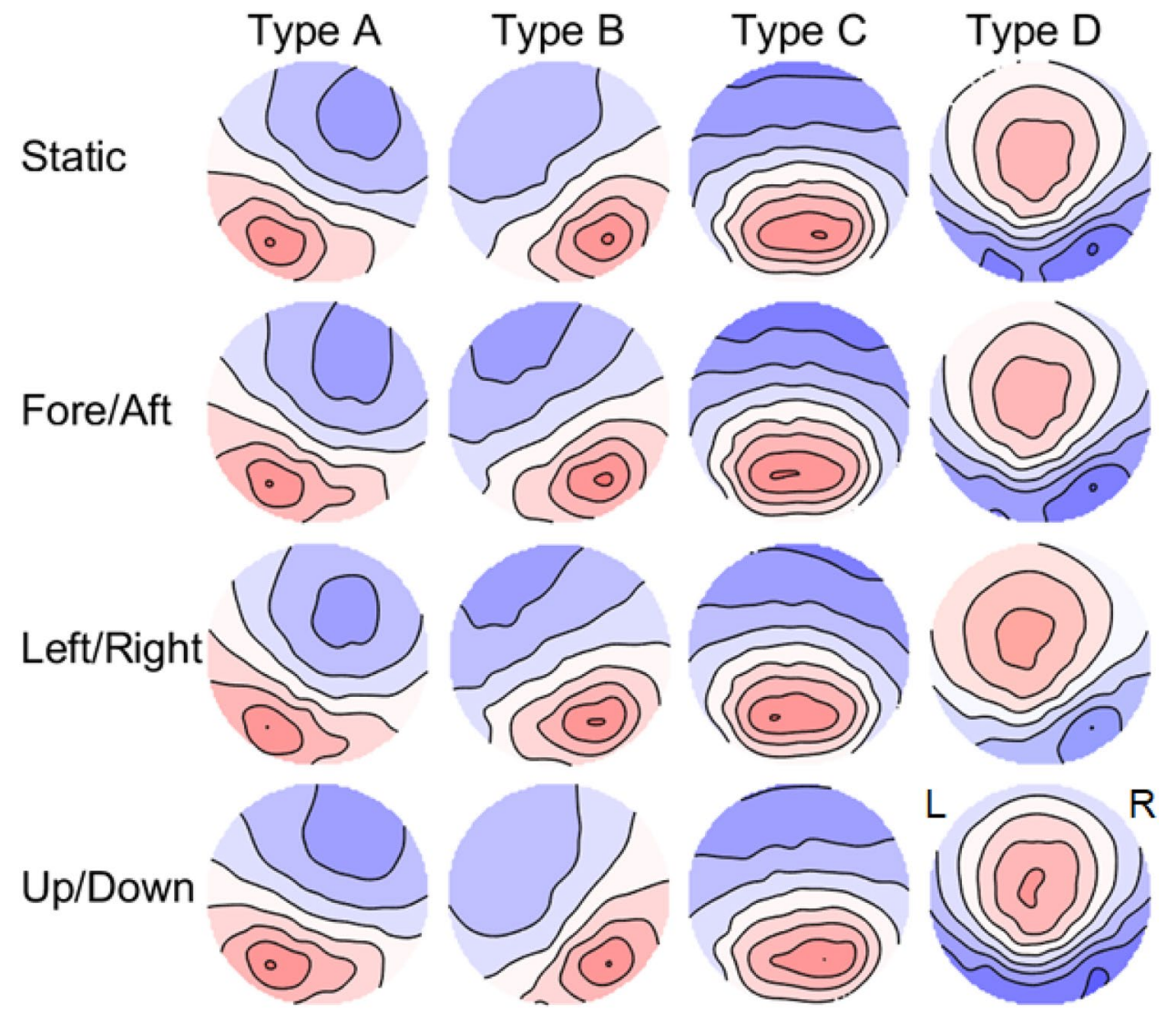

The explained variance of the microstates was $76.3 \%$ across all conditions with no significant difference between the conditions $(F(3,112)=0.37, p=0.777)$. This explained variance was well within the range (65-84\%) typically reported in microstate analyses [1]. The average mean duration, defined as the average length of time a certain microstate remains stable whenever it appears, of the microstates were A: $67.1 \mathrm{~ms}, \mathrm{~B}: 70.2 \mathrm{~ms}, \mathrm{C}: 67.7 \mathrm{~ms}$, and D: $69.6 \mathrm{~ms}$ and therefore approximately $10 \mathrm{~ms}$ shorter compared to previous reports using an eyes-closed resting condition [15].

The mean contributions are the relative portions of the total time spent in any of the four microstates. In our data, the contributions of the four states were A: $25.5 \%$, B: $24.7 \%$, C: $24.9 \%$, D: $24.9 \%$. We also analyzed the average frequency of observation of the four microstates per second, which is called occurrence. The mean occurrences were A: $3.69 / \mathrm{s}$, B: 3.46/s, C: $3.70 / \mathrm{s}$, D: 3.58/s. No significant differences between the conditions were found for any of the three metrics. Thus, our analyses showed a smaller variance between the microstates with respect to duration, occurrence, and contribution compared to previous reports [15].

In conclusion, our results show that the EEG microstate architecture is, contrary to visual stimulation, invariant with respect to weak whole-body accelerations. To date, the relevance of the temporal structure as well as the correct number of microstates, their interpretation and the relationship between EEG microstates and the resting state networks measured by fMRI are only partially understood [1-3]. Future studies on microstates might reveal valuable insights, e.g., when comparing patients suffering from vestibular failure or functional dizziness and healthy controls, or when stronger vestibular stimuli are used.

Acknowledgements Funded by the German Foundation for Neurology (DSN). We thank Katie Göttlinger for copyediting the manuscript.

Funding Open Access funding provided by Projekt DEAL.

\section{Compliance with ethical standards}

Conflicts of interests The authors declare that they have no conflict of interest.

Ethical approval This study was conducted in accordance with the Declaration of Helsinki and approved by the local ethics committee.

Open Access This article is licensed under a Creative Commons Attribution 4.0 International License, which permits use, sharing, adaptation, distribution and reproduction in any medium or format, as long as you give appropriate credit to the original author(s) and the source, provide a link to the Creative Commons licence, and indicate if changes were made. The images or other third party material in this article are included in the article's Creative Commons licence, unless indicated 
otherwise in a credit line to the material. If material is not included in the article's Creative Commons licence and your intended use is not permitted by statutory regulation or exceeds the permitted use, you will need to obtain permission directly from the copyright holder. To view a copy of this licence, visit http://creativecommons.org/licenses/by/4.0/.

\section{References}

1. Michel CM, Koenig T (2018) EEG microstates as a tool for studying the temporal dynamics of whole-brain neuronal networks: a review. Neuroimage 180:1-17. https://doi.org/10.1016/j.neuro image.2017.11.062

2. Khanna A, Pascual-Leone A, Michel CM, Farzan F (2015) Microstates in resting-state EEG: current status and future directions. Neurosci Biobehav Rev 49:105-113. https://doi.org/10.1016/j. neubiorev.2014.12.010

3. Pascual-Marqui RD, Lehmann D, Faber P et al (2014) The resting microstate networks (RMN): cortical distributions, dynamics, and frequency specific information flow. arXiv:14111949 1-14

4. Van De Ville D, Britz J, Michel CM (2010) EEG microstate sequences in healthy humans at rest reveal scale-free dynamics. Proc Natl Acad Sci USA 107:18179-18184. https://doi. org/10.1073/pnas.1007841107

5. Seitzman BA, Abell M, Bartley SC et al (2017) Cognitive manipulation of brain electric microstates. Neuroimage. https://doi. org/10.1016/j.neuroimage.2016.10.002

6. Marx E, Stephan T, Nolte A et al (2003) Eye closure in darkness animates sensory systems. Neuroimage 19:924-934. https://doi. org/10.1016/S1053-8119(03)00150-2

7. Brodoehl S, Klingner CM, Witte OW (2015) Eye closure enhances dark night perceptions. Sci Rep 5:1-10. https://doi.org/10.1038/ srep10515
8. Ertl M, Boegle R (2019) Investigating the vestibular system using modern imaging techniques-a review on the available stimulation and imaging methods. J Neurosci Methods. https://doi. org/10.1016/j.jneumeth.2019.108363

9. Ertl M, Moser M, Boegle R et al (2017) The cortical spatiotemporal correlate of otolith stimulation: vestibular evoked potentials by body translations. Neuroimage 155:50-59. https://doi. org/10.1016/j.neuroimage.2017.02.044

10. Oh SY, Boegle R, Ertl M et al (2018) Multisensory vestibular, vestibular-auditory, and auditory network effects revealed by parametric sound pressure stimulation. Neuroimage 176:354-363. https://doi.org/10.1016/j.neuroimage.2018.04.057

11. Kirsch V, Boegle R, Keeser D et al (2018) NeuroImage Handedness-dependent functional organizational patterns within the bilateral vestibular cortical network revealed by fMRI connectivity based parcellation. Neuroimage 178:224-237. https://doi. org/10.1016/j.neuroimage.2018.05.018

12. Dieterich M, Bense S, Lutz S et al (2003) Dominance for vestibular cortical function in the non-dominant hemisphere. Cereb Cortex 13:994-1007

13. Brandt T, Bartenstein P, Janek A, Dieterich M (1998) Reciprocal inhibitory visual-vestibular interaction-visual motion stimulation deactivates the parieto-insular vestibular cortex. Brain 121:1749-1758. https://doi.org/10.1093/brain/121.9.1749

14. von Wegner F, Knaut P, Laufs H (2018) EEG microstate sequences from different clustering algorithms are informationtheoretically invariant. Front Comput Neurosci 12:1-14. https:// doi.org/10.3389/fncom.2018.00070

15. Koenig T, Prichep L, Lehmann D et al (2002) Millisecond by millisecond, year by year: normative EEG microstates and developmental stages. Neuroimage 16:41-48. https://doi.org/10.1006/ nimg.2002.1070 